\title{
Queixas vocais e grau de disfonia em professoras do ensino fundamental
}

\section{Vocal complaints and degree of dysphonia in elementary school teachers}

\author{
Luciana Lemos de Azevedo', Luciana Vianello², Hellen Glayce Pereira de Oliveira ${ }^{3}$, \\ Igor de Alvarenga Oliveira ${ }^{4}$, Bruna Ferreira Valenzuela de Oliveira ${ }^{5}$, Carla Menezes da Silva ${ }^{5}$
}

\begin{abstract}
RESUMO
Objetivo: Realizar o levantamento das queixas vocais e grau de disfonia encontradas em professoras de uma Escola Municipal da Cidade de Betim. Métodos: Foi realizada coleta de dados de 13 professoras do ensino fundamental, com idade entre 20 a 50 anos (média de 36 anos) e carga horária mínima de 20 horas semanais, por meio de questionário aplicado e gravação de voz, no programa de análise acústica GRAM 5.7. Os dados foram analisados e as vozes classificadas por três fonoaudiólogas com experiência clínica na área, mínima de cinco anos. Resultados: Das professoras incluídas no estudo, 30,77\% apresentaram qualidade vocal sem alteração, 23,08\% apresentaram disfonia discreta, 46,15\% disfonia moderada e nenhuma apresentou disfonia severa. Intervalos de confiança de $95 \%$ estimaram proporções mínimas de 6,6\% de professoras com disfonia discreta e 22,39\% com disfonia moderada na população de referência. Independentemente do tipo de queixa vocal, o número total de queixas entre as professoras com disfonia moderada foi significantemente maior do que entre professoras sem alterações de voz. Não houve, no entanto, diferenças entre os grupos na distribuição das queixas específicas, quando estas foram analisadas isoladamente. Conclusão: A maioria dos professores da amostra apresentou qualidade vocal alterada, sendo o grau moderado o mais frequente. Queixas específicas parecem se distribuir igualmente entre professoras com e sem alteração de voz. Os resultados apontam a importância de projetos de saúde vocal para esta população.
\end{abstract}

Descritores: Voz; Fonação; Qualidade da voz; Distúrbios da voz; Doenças profissionais

\section{INTRODUÇÃO}

A maneira como alguns profissionais (professor, cantor, ator entre outros) utilizam a voz para se comunicar profissionalmente é conhecida como voz profissional ${ }^{(1)}$. A voz, além de ser considerada o principal instrumento de trabalho desses profissionais, é um meio de transmissão de sentimentos e conhecimentos $^{(2-3)}$.

Trabalho realizado na Pontifícia Universidade Católica de Minas Gerais PUC-Minas - Belo Horizonte (MG), Brasil.

(1) Doutora, Professora Adjunto do Curso de Fonoaudiologia da Pontifícia Universidade Católica de Minas Gerais - PUC-Minas - Belo Horizonte (MG), Brasil.

(2) Pós-graduanda do Programa Saúde do Adulto da Faculdade de Medicina da Universidade Federal de Minas Gerais - UFMG - Belo Horizonte (MG), Brasil; Professora do Curso de Fonoaudiologia da Universidade FUMEC Belo Horizonte (MG), Brasil.

(3) Especializanda em Audiologia pela Pontifícia Universidade Católica de Minas Gerais - PUC-Minas - Belo Horizonte (MG), Brasil.

(4) Especializando em Audiologia pela Pontifícia Universidade Católica de Minas Gerais - PUC-Minas - Belo Horizonte (MG), Brasil.

(5) Pós-graduanda em Estudos Linguísticos pela Universidade Federal de Minas Gerais - UFMG - Belo Horizonte (MG), Brasil

(5) Mestre, Professora do Curso de Fonoaudiologia da Pontifícia Universidade Católica de Minas Gerais - PUC-Minas - Belo Horizonte (MG), Brasil.

Endereço para correspondência: Luciana Lemos de Azevedo. Av. Brasil, 2023/2 ${ }^{\circ}$ andar, Funcionários, Belo Horizonte - MG, CEP: 30140-002. Email: azevedoll@terra.com.br

Recebido em: 28/2/2008; Aceito em: 15/11/2008
Os professores pertencem a este grupo e são os profissionais da voz mais acometidos por transtornos vocais. Dessa forma, esses profissionais frequentemente procuram o fonoaudiólogo para tratamento vocal, uma vez que é comum apresentarem alterações vocais atribuídas a condições desfavoráveis de trabalho e ao desconhecimento dos cuidados que devem ter com a voz, o que acaba provocando uma série de abusos nocivos à saúde vocal ${ }^{(4-8)}$. Os fatores negativos mais comuns aos professores envolvem presença de ruído, fumo, pó de giz, álcool, fala excessiva, poeira, beber gelado e fala em forte intensidade ${ }^{(5,9-15)}$. As queixas vocais mais frequentes são: rouquidão, fadiga vocal, pigarro, tosse, dor de garganta, ardor e garganta seca ${ }^{(5,11-16)}$. Na cidade de Belo Horizonte, um estudo realizado com os professores da Rede Municipal de Ensino evidenciou que o mau uso e o abuso vocal são os principais fatores causais de disfonia entre esses profissionais ${ }^{(17)}$.

A voz, quando produzida em intensidade e projeção adequada, proporciona um bom desempenho vocal para este profissional ${ }^{(16)}$. No entanto, vários fatores podem levar esses profissionais a cometerem abusos vocais. A acústica desfavorável, o alto índice de ruído competitivo no interior da sala de aula, a ausência de equipamentos de amplificação sonora, a necessidade de lecionar por vários turnos, a ausência de cuidados necessários à saúde vocal e a exigência de produtividade, somados ao pouco conhecimento de técnicas vocais adequadas para o exercício da profissão, são alguns desses fatores ${ }^{(5,8)}$. 
Esses abusos vocais, quando ocorrem de maneira constante, podem levar a alterações nas pregas vocais e, consequentemente, a impactos negativos na vida do professor, interferindo também na sua relação com o aluno ${ }^{(9-10,17-23)}$.

Além das consequências apresentadas anteriomente, as alterações vocais nos professores podem ainda provocar prejuízos à rede particular e pública de ensino, em decorrência do grande número de afastamentos e faltas ao trabalho ${ }^{(5,10)}$.

Segundo o $3^{\circ}$ Consenso Nacional sobre Voz Profissional de $2004^{(1)}$, as doenças relacionadas ao trato vocal, decorrentes ou prejudiciais ao trabalho provocam efeitos nos níveis social, econômico, profissional e pessoal, e representam, no Brasil, um prejuízo estimado superior a 200 milhões de reais ao ano. Em 2004, foi apresentado um documento ao Centro de Referência em Saúde do Trabalhador do Estado de São Paulo (CEREST), discorrendo sobre a determinação do papel dos fatores ambientais e organizacionais do trabalho, atuando como fatores de risco para o desenvolvimento de Distúrbio da Voz relacionado ao Trabalho e os impactos gerados na vida do trabalhor ${ }^{(24)}$. Nesse mesmo ano, foram coletados, na Gerência de Saúde do Servidor e Perícia Médica (GSPM), os dados disponíveis dos professores da Rede Municipal de Ensino de Belo Horizonte afastados do trabalho por disfonia, no período de janeiro de 1996 a maio de 2004. Foram encontrados 166 casos de readaptações funcionais definitivas em razão da disfonia ${ }^{(6)}$. Trabalhos realizados com professores da Rede Municipal de Ensino de Belo Horizonte evidenciaram que 30\% destes relataram afastamentos da sala de aula por problemas vocais durante a sua carreira ${ }^{(9-10)}$.

Em uma pesquisa realizada com 75 professores de cinco escolas do Rio Grande do Sul, a maioria (65\%) relatou perceber negativamente a sua voz, usando termos como: "horrível, rouca, complicada e enjoada"; $76 \%$ dos professores relataram possuir algum tipo de alteração vocal ${ }^{(21)}$. Outro estudo realizado com professores de creches em São Paulo reportou que 73 em 93 educadores $(80 \%)$ acreditavam ter ou ter tido disfonia ${ }^{(13)}$. $\mathrm{Na}$ Rede Municipal de Ensino de Belo Horizonte, 24 entre 48 professores triados $(50 \%)$ apresentaram voz alterada ${ }^{(14)}$.

Levando em consideração o alto índice de alterações e queixas vocais encontradas em professores e nas consequências negativas que as mesmas trazem para a vida desta população ${ }^{(2-3,5,9-11,17,20,23)}$, é de suma importância, no campo da Fonoaudiologia, a realização de estudos que investiguem ocorrência de disfonia, sua gravidade e as principais queixas vocais apresentadas. Desta forma, será possível ampliar o espectro de análise e atuação nos processos que envolvem a disfonia.

O objetivo desta pesquisa foi realizar um levantamento das queixas vocais e grau de disfonia mais frequentes em professoras do ensino fundamental de uma escola municipal da cidade de Betim (MG).

\section{MÉTODOS}

Este estudo foi aprovado pelo Comitê de Ética em Pesquisa (CEP) da instituição de origem, sob protocolo número 0085.0.213.000-06. Todas as participantes assinaram um termo de consentimento livre e esclarecido.

Foram selecionadas todas as professoras que lecionavam no ensino fundamental com, no mínimo, uma carga horária média de 20 horas semanais e, no máximo, de 40 horas semanais, de uma escola estadual de Betim (MG). Foram excluídas as professoras de Educação Física, em decorrência das particularidades de sua metodologia de ensino e outros professores que apresentaram, segundo laudo médico, algum processo inflamatório em via aérea superior, tendo em vista que tal processo normalmente interfere na qualidade vocal.

Foram sujeitos desta pesquisa todas as 13 professoras que lecionavam para o ensino fundamental em uma escola municipal da cidade de Betim (MG), que preencheram os critérios de inclusão. A idade das participantes variou entre 20 e 50 anos (média de 36 anos).

Com base na literatura foi elaborado um questionário (Anexo 1) com perguntas objetivas sobre dados pessoais, reações proprioceptivas em relação à voz e o uso da voz na escola. Cada professora recebeu o questionário, que antes de começar a ser respondido, foi lido pelo pesquisador em voz alta e as dúvidas apresentadas foram sanadas. Após a leitura, cada professora respondeu o questionário sem interferência do pesquisador.

Após a aplicação do questionário, as professoras foram submetidas à gravação da voz por meio do programa GRAM 5.7. Foram gravados trechos de fala espontânea (relato da idade, profissão e o que achavam de sua voz), sequência automática (contagem de números de 1 a 25) e emissão da vogal [a] sustentada. A voz foi gravada em ambiente acusticamente tratado, em um computador Pentium IV HP®, com sistema Windows 98 e placa de som Creative Sound Blaster. Foi utilizado um microfone unidirecional da marca Sony ${ }^{\circledR}$, modelo FV 310, devidamente conectado ao computador e situado a uma distância de cinco centímetros, lateralmente à boca das professoras.

Todas as amostras de fala (vogal sustentada, sequência automática e fala espontânea) foram gravadas em CD e analisadas por três fonoaudiólogos especialistas em voz com experiência clínica na área mínima de cinco anos. Cada fonoaudiólogo recebeu um protocolo específico (Protocolo de Análise Perceptivo-Auditiva da Voz - Anexo 2) elaborado de acordo com a literatura pesquisada ${ }^{(5,17,19,23)}$, que os direcionou na análise. Esse protocolo continha parâmetros como qualidade de voz e grau de alteração vocal. As avaliações foram realizadas individualmente pelos fonoaudiólogos, os quais ouviram por três vezes cada amostra para avaliação e conferência da avaliação; em seguida, preencheram o protocolo específico. Foi considerada a classificação mais frequente dentre os aspectos avaliados, aquela que fosse marcada por pelo menos dois fonoaudiólogos.

Além da estatística descritiva, os dados de prevalência de disfonia e queixas vocais foram analisados por meio de teste de proporções, utilizado para estabelecer se as proporções encontradas na amostra permitem inferir a presença de proporções não-nulas (maiores que zero) de indivíduos classificados nas diferentes categorias de alteração vocal. Além disso, foram construídos intervalos de confiança de $95 \%$ para as proporções. Destes intervalos, foi utilizado o valor mínimo como referência. A frequência de queixas vocais entre os diferentes grupos de gravidade de disfonia foi comparada com o teste 
Qui-quadrado de Pearson. Para todas as análises o nível de significância estabelecido foi de $0,05 \%$.

\section{RESULTADOS}

Dentre as 13 professoras estudadas, quatro $(30,77 \%)$ apresentaram qualidade vocal sem alteração e nove $(69,23 \%)$ apresentaram voz alterada: três professoras apresentaram disfonia discreta $(23,08 \%)$, seis apresentaram disfonia moderada $(46,15 \%)$ e nenhuma apresentou disfonia severa.

Foram encontradas proporções estatisticamente significantes (com $\mathrm{p} \leq 0,05)$, para professoras com voz sem alteração $(\mathrm{p}=0,00)$, disfonia discreta $(\mathrm{p}=0,00)$ e moderada $(\mathrm{p}=0,01)$. A proporção de professoras com disfonia severa não apresentou significância $(\mathrm{p}=1,00)$. Com base nas proporções encontradas, é possível estimar com $95 \%$ de confiança, que um mínimo de 11,26\% de professoras apresentarão voz normal, 6,6\% apresentarão disfonia discreta e 22,39\% apresentarão disfonia moderada, na população de referência.

Independente da existência de alteração vocal, as professoras apresentaram várias queixas vocais. Todas as queixas vocais relatadas, assim como a significância do teste de proporções para a identificação de proporções estatisticamente significantes estão listadas na Tabela 1.

A comparação entre a proporção de queixas apresentadas pelos grupos de professoras com voz normal, disfonia discreta

Tabela 1. Distribuição numérica e percentual da ocorrência de queixas vocais auto referida pelos professores

\begin{tabular}{lcc}
\hline Tipo de queixa & $\begin{array}{c}\text { Professoras que } \\
\text { apresentaram queixas }\end{array}$ & Valor de $p$ \\
\hline Coceira & 2 & 0,13 \\
Ardência & 3 & $0,00^{\star}$ \\
Tensão & 2 & 0,13 \\
Dor & 3 & $0,00^{\star}$ \\
Secura & 7 & $0,00^{\star}$ \\
Tosse & 6 & $0,00^{\star}$ \\
Fadiga vocal & 7 & $0,00^{\star}$ \\
Sensação de aperto & 1 & 0,12 \\
Corpo estranho & 0 & 1,00 \\
Rouquidão & 6 & $0,00^{\star}$ \\
Outros & 2 & 0,13 \\
\hline
\end{tabular}

* Resultados estatisticamente significantes.Teste de proporção. e moderada revelou que a quantidade de queixas como um todo foi significantemente superior nas professoras com disfonia moderada, comparadas com professoras com voz normal $(\mathrm{p}=0,00)$. Não houve diferença estatisticamente significante quando comparadas as proporções de queixas entre professoras com disfonia discreta e normal $(\mathrm{p}=0,59)$ ou professoras com disfonia discreta e moderada $(\mathrm{p}=0,59)$.

A análise isolada de queixas específicas não revelou associação com o grau de disfonia, ou seja, a distribuição das proporções de cada tipo de queixa entre os grupos de voz normal, disfonia discreta e moderada não revelou diferenças significantes. Os valores de significância para a distribuição de cada tipo de queixa entre os diferentes grupos de grau de alteração vocal estão listados na Tabela 2.

\section{DISCUSSÃO}

Após análise dos resultados foi observado que o número de professoras com alteração vocal foi significantemente maior do que o sem alteração vocal. Esse dado está de acordo com outros estudos que também mostram maior incidência de professores com voz alterada em relação aos que apresentam voz sem alteração ${ }^{(9,11-14,23,25)}$. A ocorrência de professores disfônicos nesses outros estudos apresenta valores superiores ${ }^{(9,11-14,23)}$ e inferiores ${ }^{(25)}$ aos do presente estudo, sendo encontrados valores em torno de 60 a $80 \%$. Esse fato pode ser justificado pela maioria das pesquisas trazerem dados obtidos por meio de questionários, baseando-se apenas na percepção do professor sobre sua voz e não na avaliação de um especialista. No entanto, pode-se considerar que a disfonia é um problema presente na vida dos professores e, segundo a literatura, pode interferir de maneira negativa no desempenho profissional e na qualidade de vida dos mesmos ${ }^{(3,6,9-10,16,19,21-23)}$.

$\mathrm{O}$ grau de disfonia apresentado pelas professoras foi discreto e moderado. O número de professoras, da amostra estudada, com disfonia moderada é superior ao número de professoras com qualidade vocal normal ou disfonia discreta, provavelmente em função de uma possível evolução do quadro de disfonia, causado por abusos vocais constantes ${ }^{(3-8,18)}$. Esse achado reforça a hipótese de uma possível tolerância desses profissionais para usar a voz mesmo durante um quadro de disfonia $^{(3,11,19)}$. Isto confirma a falta de conhecimento de técnicas vocais adequadas e a necessidade da realização de programas de prevenção junto a estes profissionais, a fim de orientá-los quanto ao uso e saúde vocal ${ }^{(3,6,9,18-21)}$.

A partir da soma da proporção de professoras com dis-

Tabela 2. Comparação entre o tipo de queixa vocal e a qualidade vocal

\begin{tabular}{|c|c|c|c|c|c|c|c|c|c|c|c|c|c|c|c|c|c|c|}
\hline \multirow[t]{2}{*}{ Qualidade vocal } & \multicolumn{2}{|c|}{$\begin{array}{l}\text { Queixa de } \\
\text { ardência }\end{array}$} & \multicolumn{2}{|c|}{$\begin{array}{l}\text { Queixa } \\
\text { de dor }\end{array}$} & \multicolumn{2}{|c|}{$\begin{array}{c}\text { Queixa } \\
\text { de secura }\end{array}$} & \multicolumn{2}{|c|}{$\begin{array}{l}\text { Queixa } \\
\text { de tosse }\end{array}$} & \multicolumn{2}{|c|}{$\begin{array}{l}\text { Queixa de } \\
\text { fadiga vocal }\end{array}$} & \multicolumn{2}{|c|}{$\begin{array}{l}\text { Queixa de } \\
\text { rouquidão }\end{array}$} & \multicolumn{2}{|c|}{$\begin{array}{c}\text { Queixa } \\
\text { de coceira }\end{array}$} & \multicolumn{2}{|c|}{$\begin{array}{c}\text { Queixa } \\
\text { de tensão }\end{array}$} & \multicolumn{2}{|c|}{$\begin{array}{c}\text { Queixa de sensação } \\
\text { de aperto }\end{array}$} \\
\hline & Não & Sim & Não & Sim & Não & Sim & Não & Sim & Não & Sim & Não & Sim & Não & Sim & Não & Sim & Não & Sim \\
\hline Normal & 3 & 1 & 4 & 0 & 2 & 2 & 2 & 2 & 2 & 2 & 3 & 1 & 3 & 1 & 3 & 1 & 4 & 0 \\
\hline Disfonia discreta & 3 & 0 & 1 & 2 & 1 & 2 & 2 & 1 & 1 & 2 & 2 & 1 & 3 & 0 & 3 & 0 & 3 & 0 \\
\hline Disfonia moderada & 4 & 2 & 5 & 1 & 3 & 3 & 3 & 3 & 3 & 3 & 2 & 4 & 5 & 1 & 5 & 1 & 5 & 1 \\
\hline Valor de $p$ & \multicolumn{2}{|c|}{0,25} & \multicolumn{2}{|c|}{0,78} & \multicolumn{2}{|c|}{0,87} & \multicolumn{2}{|c|}{0,87} & \multicolumn{2}{|c|}{0,87} & \multicolumn{2}{|c|}{0,38} & \multicolumn{2}{|c|}{0,88} & \multicolumn{2}{|c|}{0,68} & \multicolumn{2}{|c|}{0,35} \\
\hline
\end{tabular}

Teste Qui-quadrado de Pearson 
fonia discreta e disfonia moderada, considerando a margem de erro, sugere-se que o número de professoras com disfonia provavelmente será mais do que o dobro de professoras que apresentam voz sem alterações. Isto reafirma o alto índice de professores com qualidade vocal alterada ${ }^{(5,9,18-23)}$.

Independentemente do grau de alteração vocal, as professoras apresentaram queixas vocais. As principais queixas vocais observadas (secura, fadiga vocal, tosse, rouquidão, dor e ardência) também foram observadas em outros estudos ${ }^{(5,11-15,18-19)} \mathrm{em}$ proporções aproximadas em relação ao presente estudo. Tais achados foram obtidos por meio de questionário em ambos os estudos, sendo considerada a percepção do professor.

Dentre essas queixas, as mais frequentes foram: secura e fadiga vocal. Em outro estudo, a fadiga vocal também foi citada como uma das queixas mais frequentes $(72,6 \%)$, estando abaixo apenas da rouquidão $(81 \%)$. Essas queixas podem estar relacionadas ao possível desconhecimento de técnicas vocais adequadas para lecionar, falta de hidratação vocal, tensão ao falar e condições de trabalho desfavoráveis como forte ruído competitivo, salas com padrão acústico desfavorável, dentre $\operatorname{outros}^{(3,5-10,18)}$, sendo também indício de mau uso ou abuso vocal.

Para os casos de queixas que foram mais frequentes, elas parecem estar distribuídas igualmente entre as professoras com qualidade vocal sem alterações e as que apresentam disfonia de grau discreto e moderado. Observa-se então, que indepen- dente da presença de disfonia, as professoras, devido à falta de informação, cometem muitos abusos vocais, tendo como consequência as queixas e alterações vocais ${ }^{(3,17-21)}$.

Os achados do presente estudo permitiram inferir o quanto é necessária a realização de trabalhos preventivos com o intuito de conscientizar e educar esta população a respeito do uso da $\operatorname{voz}^{(3,5,10-11,19)}$.

É importante ressaltar que os achados deste estudo referemse a uma escola específica, não podendo ser generalizados. No entanto, os mesmos corroboram os resultados de outras pesquisas, reforçando um alto índice de queixas e alterações vocais, demonstrando que os professores cometem abusos vocais durante seu trabalho.

Sugere-se a realização de estudos futuros com uma amostra maior e com professores de várias escolas a fim de se obter resultados mais fidedignos e que possam ser generalizados para toda a população de professores.

\section{CONCLUSÃO}

A partir do presente estudo foi possível observar que as queixas vocais mais frequentes e significativas nas professoras estudadas foram: ardência, dor, secura, tosse, fadiga, rouquidão. Além disso, a maioria das professoras apresentou qualidade vocal alterada, sendo o grau de alteração moderado o mais frequente.

\begin{abstract}
Purpose: The aim of this study was to survey vocal complaints and degree of dysphonia among teachers from a public elementary school at Betim, MG (Brazil). Methods: Thirteen elementary school teachers with ages between 20 and 50 years (mean of 36 years old), who taught at least 20 hours a week, participated in the study. Data were obtained using a questionnaire and voice recording, analyzed on the GRAM 5.7 acoustic analysis program. After acoustic analysis, the voices were perceptually analyzed and classified by three speech therapists with at least five years of clinical experience in the area. Results: From the selected teachers, 30.77\% showed normal voice quality, while $23.08 \%$ were considered to have mild dysphonia and $46.15 \%$ moderate dysphonia. None of the subjects showed severe dysphonia. Confidence intervals of $95 \%$ provided estimations of a minimum of $6.6 \%$ of teachers with discrete dysphonia and $22.39 \%$ with moderate dysphonia in the population of reference. Regardless of the type of vocal complaint, the total number of complaints among teachers with moderate dysphonia was significantly higher than among teachers with normal voice quality. Nevertheless, there were no differences between groups in the distribution of specific complaints, when these were individually analyzed. Conclusion: The majority of the teachers in this study had altered vocal quality, mostly being classified with moderated degree dysphonia. Specific vocal complaints appear to be equally distributed among teachers with normal voice and with dysphonia. Results point out the importance of vocal health projects directed to this population.
\end{abstract}

Keywords: Voice; Phonation; Voice quality; Voice disorders; Occupational diseases

\title{
REFERÊNCIAS
}

1. Voz e trabalho: uma questão de saúde e direito do trabalhador. Rio de Janeiro: Academia Brasileira de Laringologia e Voz: Associação Brasileira de Otorrinolaringologia e Cirurgia Cérvio-Facial; 2004. [3 ${ }^{\circ}$ Consenso Nacional sobre Voz Profissional].

2. Aydos BRS, Motta L, Teixeira BS. Eficácia da hidratação na redução de queixas vocais de professores. J Bras Fonoaudiol. 2000;1(2):10-5.

3. Grillo MHMM, Penteado RZ. Impacto da voz na qualidade de vida de professore(a)s do ensino fundamental. Pró-Fono. 2005;17(3):321-30.
4. Azevedo LL, Valentino J, Rodrigues ES. Repercussão do uso da voz amplificada em sala de aula. XII Congresso Brasileiro de Fonoaudiologia e II Congresso Sulbrasileiro de Fonoaudiologia; 2004; Foz do Iguaçu. Rev Soc Bras Fonoaudiol. 2004; Supl Esp.

5. Viola IC, Ferreira LP, Sene CD, Villas Boas DC, Souza SM. A voz do professor: levantamento das publicações brasileiras. Rev Soc Bras Fonoaudiol. 2000;5(7):36-45.

6. Assunção AA, Medeiros AM, Braga MLF, Barreto SM. A prevalência 
de disfonia entre os professores em readaptação funcional definitiva da rede municipal de ensino de Belo Horizonte. II Simpósio sobre Trabalho e Educação; 2004; UFMG. Belo Horizonte: Anais. 2004.

7. Ferro L, Navarrete RG, Rocha SD. A importância da hidratação vocal para o docente. J Bras Fonoaudiol. 2000;5(7):79-87.

8. Azevedo LL, Cicero F, Soares LAM, Andrade MM, Bahia R, Cristina Y. Triagem vocal de professores de ensino superior. XIII Congresso Brasileiro de Fonoaudiologia, 2005, Santos. Rev Soc Bras Fonoaudiol. 2005; Supl Esp.

9. de Medeiros AM, Barreto SM, Assunção AA. Voice disorders (dysphonia) in public school female teachers working in Belo Horizonte: prevalence and associated factors. J Voice. 2008;22(6):676-87.

10. Jardim R, Barreto SM, Assunção AA. Condições de trabalho, qualidade de vida e disfonia entre docentes. Cad Saúde Pública = Rep Public Health. 2007;23(10):2439-61.

11. Lemos S, Rumel D. Ocorrência de disfonia em professores de escolas públicas da rede municipal de ensino de Criciúma-SC. Rev Bras Saúde Ocup. 2005;30(112):7-13.

12. Fuess VLR, Lorenz MC. Disfonia em professores do ensino municipal: prevalência e fatores de risco. Rev Bras Otorrinolaringol. 2003;69(6):807-12.

13. Simões M, Latorre MRD, Bitar MR. Uso profissional da voz por educadores de creches: achados preliminares. Rev Soc Bras Fonoaudiol. 2000;5(7):28-35.

14. Azevedo LL, Galvão AP, Costa LP. Incidência de alterações vocais nos professores da Rede Municipal de Ensino de Belo Horizonte. VIII Congresso Brasileiro de Fonoaudiologia; 2000; Recife. Anais. Recife; 2000.

15. Gama ACC. Voz profissional: atuação fonoaudiológica no trabalho com professores e telejornalistas. In: Britto ATBO, organizador. Livro de fonoaudiologia. São José dos Campos: Pulso Edicorial; c2005. p 42533.
16. Carelli EG, Nakao M. Educação vocal na formação do docente. Fono Atual. 2002;5(22):40-52.

17. Azevedo LL, Costa LP, Galvão AP. Classificação das disfonias nos professores da Rede Municipal de Ensino de Belo Horizonte. VIII Congresso Brasileiro de Fonoaudiologia; 2000; Recife. Anais. Recife; 2000.

18. Giannini SPP, Passos MC. Histórias que fazem sentidos: as determinações das alterações vocais do professor. Distúrb Comum. 2006;18(2):245-57.

19. Penteado RZ, Bicudo Pereira IMT. Qualidade de vida e saúde vocal de professores. Rev Saúde Pública = J Public Health. 2007;41(2):236-43.

20. Penteado, RZ; Bicudo Pereira IMT. Avaliação do impacto da voz na qualidade de vida de professores. Rev Soc Bras Fonoaudiol. 2003;8(2):19-28.

21. Schwarz K, Cielo CA. A voz e as condições de trabalho de professores de cidades pequenas do Rio Grande do Sul. Rev Soc Bras Fonoaudiol. 2005;10(2):83-90.

22. Quintairos S. Incidência de nódulos vocais em professores de pré-escola e o seu tratamento. Rev CEFAC. 2000;2(1):16-22.

23. Dragone MLS. Novos caminhos para os estudos sobre a voz do professor. Fonoaudiol Brasil. 2001:1(1):43-50.

24. Centro de Referência em Saúde do Trabalhador, Coordenadoria de Controle de Doenças Secretaria de Estado da Saúde de São Paulo. Distúrbios de voz relacionados ao trabalho. Bol Epidemiol Paul. 2006;3(26):16-23.

25. Ferreira LP, Giannini SPP, Figueira S, Silva EE, Karmann DF, Souza TMT. Condições de produção vocal de professores da Prefeitura do Município de São Paulo. Distúrb Comun. 2003;14(2):275-307.

Anexo 1. Questionário

1) Identificação:

- Nome:

- Idade:

- Data de nascimento:

- Telefone:

2) Dados importantes:

Tempo que leciona: Leciona em mais de 1 escola? ( ) Sim - ( ) Não

- Média de carga horária total de regência:

- Média de carga horária de regência semanal:

horas/dia

horas/semana

3) Reações proprioceptivas (o que você sente em relação a sua voz):
( ) coceira
( ) ardência
( ) tensão
( ) dor ( ) secura
( ) fadiga vocal ( ) sensação de aperto ( ) corpo estranho ( ) rouquidão
( ) outros:

4) Sua voz fica pior quando?
( ) início do dia
( ) fim do dia
( ) a noite
( ) fim de semana

Anexo 2. Protocolo de análise perceptivo-auditiva da voz

Fonoaudiólogo:

Professora:

- Qualidade vocal: ( ) normal

( ) alterada

- Grau de alteração: ( ) discreto ( ) moderado ( ) severo ( ) extremo 